\title{
Qualidade em serviços públicos: os desafios da atenção primária
}

\author{
Quality in public services: the challenges of primary care \\ servicios públicos: los desafíos de la atención primaria \\ Leonardo Cançado Monteiro Savassi ${ }^{1 *}$
}

Palavras-chave:

Serviços de Saúde Atenção Primária à Saúde Gestão da Qualidade Cultura Organizacional Avaliação

Keywords: Health Services Primary Health Care Quality Management Organizational Culture

Evaluation

\section{Resumo}

0 Ministério da Saúde brasileiro sinalizou uma política de avaliação da qualidade da Atenção Primária através da portaria 1.654, de 19/07/2011. Toda mudança organizacional interfere na cultura de qualquer instituição, especialmente quando esta mudança está direcionada para 0 tema da qualidade. Ela envolve processos, meios internos e pessoas, e não pode ser artificialmente produzida pela força da caneta. No setor saúde, a gestão baseada na qualidade envolve mudanças de foco de atuação e de tecnologias, apoiadas no resgate da relação profissional-paciente, na forma de remuneração de médicos e equipes, na qualificação e capacitação dos profissionais, na qualificação da própria gestão e na participação ativa dos profissionais da saúde na pactuação destas metas e parâmetros de qualidade. Outros instrumentos de avaliação da qualidade já foram implantados no âmbito da Atenção Primária no subsistema público de saúde, e seu seguimento foi interrompido. Qualificar a Atenção Primária passa não apenas pela avaliação, mas pela seleção de profissionais capacitados, qualificação da prestação de serviços, monitorização de resultados e valorização dos profissionais de saúde envolvidos com a nova cultura.

\section{Abstract}

The Brazilian Ministry of Health has signaled a policy of evaluating the quality of primary care through the 1654 decree of 19.07.2011. Any organizational change interferes with the culture of any institution, especially when this change is directed toward the issue of quality. It involves processes, internal resources and people, and cannot be artificially produced by the power of the pen. In the health sector, management based on quality involves changes in business focus and technology, supported the rescue of professional-patient relationship, in the form of compensation of physicians and teams, in the qualification and training of professional, in qualifications in their own management, and in active participation of health professionals in the Agreement on these goals and quality parameters. Other instruments of quality assessment have been established within the Primary Care in public health sub-system, and its follow-up was interrupted. Qualifying the Primary is not just about evaluation, but also the selection of qualified professionals, qualification of services, monitoring results and valuation of health professionals involved with the new culture.

\footnotetext{
${ }_{1}^{1}$ Universidade Federal de Ouro Preto (UFOP). leosavassi@gmail.com

Conflito de interesses: Declaro ter recebido subvenção do Governo Federal através de diárias e passagens para participação em eventos como palestrante.

*Autor correspondente.

Fonte de financiamento: nenhuma

Conflito de interesses: declara não haver.

Recebido em: 05/08/2011

Aprovado em: 16/04/2012
} 


\section{Palabras clave: \\ Servicios de Salud \\ Atención Primaria de Salud \\ Gestión de Calidad \\ Cultura Organizaciona}

Evaluación

\section{Resumen}

El Ministerio de Salud de Brasil ha dado señales de una política de evaluación de la calidad de la atención primaria a través del decreto 1654 de 19.07.2011. Cualquier cambio en la organización interfiere con la cultura de cualquier institución, sobre todo cuando este cambio se dirige hacia el tema de la calidad. Implica en procesos internos, recursos y personas, y no puede ser producido artificialmente por el poder de la pluma. En el sector de la salud, la administración basada en la gestión de calidad implica en cambios en el enfoque de negocio y la tecnología apoyada en lo rescate de la relación profesional-paciente, en la forma de compensación de equipos y médicos, en la cualificación y formación de los profesionales, en la cualificación en su propia gestión y en la participación activa de profesionales de la salud en el acuerdo sobre los objetivos y parámetros de calidad. Otros instrumentos de evaluación de la calidad se han establecido dentro de la Atención Primaria en el sistema de salud sub-público, y su seguimiento se interrumpió. La cualificación de la atención primaria no ocurre sólo por la evaluación, pero también por la selección de profesionales cualificados, la calificación de servicios, lo seguimiento de los resultados y la valoración de los profesionales de la salud involucrados con la nueva cultura.

\section{Introdução}

A globalização e a busca pela qualidade afetaram a postura do consumidor, exigindo desempenho confiável de produtos e serviços, sem tolerância de tempo ou falhas. A qualidade foi o agente de mudança corporativa mais importante do novo milênio e é a demanda gerencial mais importante a ser enfrentada pelas instituiçóes ${ }^{1}$.

O setor de serviços liderou a expansão econômica e o crescimento global, e a preocupação inicial com a qualidade dos produtos foi ampliada para a qualidade de serviços e comportamento humano, colocando o ser humano e a qualidade pessoal em primeiro lugar ${ }^{2}$.

No mercado de saúde, a busca pela qualidade náo é diferente: clientes cada vez mais exigentes e menos tolerantes querem acesso a médicos, procedimentos e exames imediatamente, pois não há tempo para despender com sua saúde. Assim, o conceito de qualidade hoje está muito mais próximo do que os clientes julgam que seja, do que propriamente a visão do gestor sobre o seu serviço ${ }^{3}$.

Os serviços de saúde de alta qualidade estão preocupados náo somente com a adequaçáo dos serviços para o diagnóstico e manejo de doenças, mas também com a adequação dos serviços que previnem doenças futuras e promovem melhoras na saúde. (STARFIELD, 2002, p. 419$)^{4}$.

O setor saúde está submetido a uma forte pressão por mudanças, devido ao esgotamento do modelo de pagamento por procedimentos (Saúde suplementar), não reconhecimento da performance profissional (Saúdes pública e suplementar) e deterioração da relação com o paciente. A área de saúde enfrenta também outras crises, como as de mão de obra qualificada, de limitaçôes orçamentárias e até da real vocação institucional do serviço de saúde ${ }^{3}$.
No subsetor Suplementar, processos de certificação de qualidade e de acreditação estão pautados na busca pelos certificados da Organização Internacional de Normalização (International Organization for Standardization), ISO 9000, e pela Organização Nacional de Acreditação (ONA), exemplos de iniciativas voltadas para reconhecer a melhoria da qualidade.

No subsetor público do Sistema Único de Saúde (SUS), há iniciativas como o processo AMQ (Avaliação para Melhoria da Qualidade) voltado para a requalificação da Atenção Primária, além de vários instrumentos de planejamento como a Programação Pactuada e Integrada (PPI), o Termo de Ajuste de Metas (TAM) e o Pacto dos Indicadores da Atenção Básica em Saúde 5 .

O Governo Federal lançou em julho de 2011 o Programa Nacional de Melhoria do Acesso e da Qualidade da Atenção Básica (PMAQ-AB), uma atitude louvável desde que adequada a indicadores factíveis e acoplada a uma mudança no paradigma assistencial, que envolve tanto a escolha de parâmetros adequados para a avaliação quanto o investimento do próprio governo na qualidade dos profissionais estando ainda vinculado à mudança da organização do próprio sistema de saúde brasileiro como um todo, e não somente de uma parte dele ${ }^{6}$.

O objetivo deste manuscrito é debater abertamente as políticas de avaliação da qualidade de serviços de saúde, com foco na Atenção Primária à Saúde.

\section{Metodologia}

Desenvolvimento de uma discussão sobre a qualidade em serviços de saúde a partir de revisão narrativa sobre as portarias ministeriais que definem instrumentos de avaliação da qualidade dos serviços públicos, com foco na atenção 
primária. Foram revisadas as portarias que definem tais instrumentos a partir da implantação do então Programa Saúde da Família em 1994.

\section{Resultados e discussão}

Os serviços de saúde devem ser seguros (evitar danos ao usuário), efetivos (prestados com base na melhor evidência científica), oportunos (esperas e deslocamentos mínimos), centrados nos usuários (atendam às necessidades e demandas deles) e humanizados (relação profissional-usuário empática num ambiente acolhedor $)^{4,7}$.

No setor saúde, dois públicos-alvo são foco de qualquer ação que envolva avaliação da qualidade: os clientes internos - setor de pessoal, departamentos de gestáo e profissionais do serviço -; e os clientes externos - os próprios "pacientes", familiares e serviços complementares.

Gerir a qualidade em organizaçóes públicas de saúde é um desafio. Mais do que uma mudança gerencial ou de financiamento, é necessário reorganizar a cadeia produtiva com novos conceitos e quebrar paradigmas assistenciais que alterem a cultura organizacional no sentido da qualidade no atendimento. E o novo paradigma mundial dos sistemas de saúde aponta a Atenção Primária como reestruturante.

A saúde brasileira baseou-se no modelo predominante nos Estados Unidos, de pagamento por procedimentos, valorização da subespecialização, seguridade social vinculada a seguros de saúde e priorização de tecnologias duras. O desenvolvimento dos serviços de saúde brasileiros foi heterogêneo, com diferentes lógicas: o modelo campanhista do início do século; o modelo das Santas Casas de Misericórdia; o modelo asilar estatal (especialmente em tuberculose, psiquiatria e hanseníase); o modelo previdenciário (Bismarkiano); o modelo sanitarista; até o modelo atual de sistemas nacionais de saúde (Beveridgiano) ${ }^{8}$.

Além disto, em quaisquer etapas deste desenvolvimento, os sistemas de saúde foram desenhados para atender condiçóes agudas (especialmente infecciosas), enquanto a transição epidemiológica mudou o perfil do usuário destes serviços, que passou a ser consumidor de tecnologias determinadas por condiçóes crônicas?

O dilema da conformação dos serviços de saúde para o enfrentamento das condiçóes agudas e a necessidade de resposta concomitante às causas externas e prioritariamente às condiçóes crônicas persiste. A resposta da "medicina preventiva" baseia-se em evidências nem sempre científicas, sob variabilidade de condutas e gastos desproporcionados, bem como iatrogenia para o paciente, e a "promoçáo da saúde" ainda é ínfima, baseada no carro-chefe das academias de saúde pública ${ }^{10}$.

Os serviços públicos baseiam-se em estabilidade por concurso público, o que gera outros dilemas: planos de carreira impactam na folha de pagamento, os salários iniciais são baixos, e as instituiçôes são forçadas a criar adicionais não incorporados ao salário base. Há um achatamento entre o piso salarial e o teto máximo atingido, que gera acomodação, e os salários inferiores ao subsetor suplementar fazem com que o serviço público não seja a fonte principal de renda dos profissionais, em especial o médico.

Da mesma maneira, como o serviço público é remunerado por cargos ou carreira, não encontra meios de privilegiar ou beneficiar o profissional que exerce sua profissão de acordo com as necessidades dos usuários, com parâmetros de qualidade ou que se integre à cultura das organizaçôes públicas. Bons e maus profissionais recebem igualmente pela carga horária trabalhada, o que na prática privilegia o profissional ruim ou aquele que se pauta pelo menor esforço. MENDES $(2008)^{7}$ aponta alguns paradigmas, princípios e leis de mercado que definem dificuldades a se enfrentar na tarefa de reorganização do setor saúde (Quadro 1) 9,11-13.

$\mathrm{Na}$ atenção primária, a satisfação do usuário é superior quando baseada na estratégia saúde da família ${ }^{14}$, e a satisfação de quem utiliza a porção assistencial do SUS é maior que a de quem só conhece sua assistência pelos telejornais ${ }^{15}$. A atenção primária e/ou ambulatorial, embora mais flexível e menos hierárquica, também se baseia na cooperação, com um forte componente de trabalho em equipe, necessário para a prestação de serviços adequados e sucesso das intervençóes.

$\mathrm{O}$ trabalho em equipe fragiliza-se à medida que a atenção primária se torna uma fonte de "pontos para ingressar em programas de residência" ${ }^{16}$, considera recém-formados com horizonte profissional indefinido similares a Médicos de Família e Comunidade e fragmenta a equipe com a desvinculação do médico e divisão de dois médicos por equipe, bem como três para duas e até quatro médicos para três equipes ${ }^{17}$. Não valorizar a formação específica para a Atenção Primária significa não reconhecer a qualificação dos clientes internos do SUS e, em última análise, não qualificar a própria atenção à saúde.

A reestruturação pautada pela qualidade tem vários pontos de mudança, alguns deles de difícil governabilidade por parte do gestor, outros nem tanto: ambiência (estrutura aprazível, agradável e avançada; organização no atendimento); tecnologias (leves e duras) adequadas; imagem de confiabilidade e competência técnica dos médicos e equipe; especialidades adequadas e seus conhecimentos específicos; higiene; agilidade do primeiro atendimento, na recepção/acolhimento, até a saída da unidade; definição concreta das funçôes e obrigações de cada setor e funcionários; presteza para a viabilização 
desses objetivos, priorizando sempre a satisfação do cliente. Donabedian $(1990)^{18}$ denominou de sete pilares da Qualidade em saúde, descritos no Quadro 2.

Várias foram as tentativas de se qualificar a atençáo no Sistema Único de Saúde, baseadas em ferramentas de planejamento. Um instrumento de monitoramento e avaliação de atividades na Atenção Básica foi a "Sala de Situação": uma ferramenta de diagnóstico situacional de saúde, específica para equipes de Saúde da Família, com uma série de indicadores apresentando sua situação da unidade, em especial de demanda versus oferta (dados demográficos, estrutura de saúde e profissionais envolvidos) e dados de produtividade (internaçóes, consultas, visitas domiciliares e exames complementares). Em cada quadro havia espaço para classificar a situaçáo: vermelha (situação ruim), amarela (sinal de alerta) e verde (situação boa) ${ }^{19}$.

O processo de avaliaçáo da qualidade através da Sala de Situação foi aplicado em diversos âmbitos, dentre eles o da gestão municipal e estadual, pela Rede Nacional de Informação em Saúde ${ }^{20}$. Assim, o objetivo para as Unidades Básicas de Saúde, mais do que utilizar a sala de situaçáo de saúde, era municiar o gestor municipal de dados, consolidando informaçóes locais. A diferença entre consolidar os dados da sala de situação e preencher os bancos de dados que a alimentam é que, tendo um quadro concretizado de atividades, percebia-se com mais clareza a situação (de acordo com a qualidade das equipes) com um parâmetro de qualidade a ser alcançado.

Outro instrumento, proposto pelo Projeto de Expansão e Consolidação da Saúde da Família (PROESF) em cooperação técnica com a Organização Pan-Americana de Saúde (OPAS) foi a Avaliação para Melhoria da Qualidade (AMQ) ${ }^{21}$. O AMQ foi desenvolvido especificamente para a Atenção Básica, com foco na autonomia de gestáo do processo de trabalho pelas equipes, através de parâmetros inclusos em cinco instrumentos de autoavaliação. Lançado em 2005, a lógica do AMQ envolvia uma série de parâmetros analisados em um momento inicial (chamado AMQ-zero), nos quais a equipe se classificava e, a partir disto, trabalhava para conseguir subir ao menos um nível nos parâmetros em questão até a avaliação subsequente (AMQ-1).

No ano seguinte, foi lançado o software Programação da Atenção Básica (Prograb), um instrumento de planejamento voltado para a organização da agenda de trabalho dos profissionais de saúde que continha parâmetros de atividades das Equipes de Saúde da Família que guardam similaridades com os 47 parâmetros prioritários definidos na PMAQ-AB ${ }^{22}$.

Uma iniciativa mais recente de avaliação da Atenção Primária, também apoiada pelo Ministério da Saúde, foi lançada em 2010: validou-se para a língua portuguesa o instrumento PCA-Tool (Ferramenta de Avaliação da Atenção Primária)

Quadro 1. Leis e princípios gerenciais aplicáveis aos serviços de saúde.

\begin{tabular}{|c|c|}
\hline \multirow{2}{*}{ Lei de Wildavsky } & 0 custo em saúde sempre se elevará até o nível máximo de recursos disponíveis (também conhecida \\
\hline & como "lei do saco sem fundo"). \\
\hline Princípio da variabilidade na prestação dos serviços de saúde & Quanto maior a variabilidade de condutas diante de um mesmo problema, maiores os custos. \\
\hline Lei de Roemer & $\begin{array}{l}\text { "se há leitos hospitalares, eles tendem a ser usados", independentemente das necessidades da } \\
\text { população. }\end{array}$ \\
\hline Princípio da indução da demanda pela oferta & $\begin{array}{l}\text { Nos sistemas de saúde, não é a demanda que regula a oferta; o serviço disponibilizado será consumido } \\
\text { até que se esgote sua capacidade (similar a Lei de Roemer). }\end{array}$ \\
\hline A Lei da Caneta do Médico & 0 carimbo médico é responsável por cerca de $80 \%$ dos gastos com saúde. \\
\hline $\begin{array}{l}\text { A Lei da Concentração da Gravidade e dos Gastos com as } \\
\text { Doenças }\end{array}$ & $\begin{array}{l}\text { Quanto mais graves e complexas as doenças, proporcionalmente maiores são os custos, e poucos } \\
\text { pacientes consomem grande parte dos recursos humanos, técnicos e financeiros em saúde. }\end{array}$ \\
\hline Lei de Hart & $\begin{array}{l}\text { Os mecanismos que interferem na oferta de serviços fazem com que os recursos sejam distribuídos } \\
\text { inversamente às necessidades. } 0 \text { uso de serviços de saúde ocorre por necessidades "percebidas" e } \\
\text { comportamento frente a seus problemas de saúde. }\end{array}$ \\
\hline
\end{tabular}

Fonte: Mendes (2008)9, Zucchi et al. (2000)11, Tavares-Júnior (2009)12 e Mendes (2010),11,12,13.

Quadro 2. Os sete pilares da Qualidade em Saúde segundo Donabedian (1990).

\begin{tabular}{|ll|}
\hline Eficácia & Capacidade para o cuidado, na melhor forma possível de contribuir para a melhoria das condições de saúde; \\
\hline Efetividade & 0 quanto melhorias possíveis nas condições de saúde são de fato obtidas; \\
\hline Eficiência & A capacidade de obter a maior melhoria possível nas condições de saúde ao menor custo possível; \\
\hline Otimização & A mais favorável relação entre custos e benefícios; \\
\hline Aceitabilidade & Conformidade com as preferências do paciente (acessibilidade, relação médico paciente, amenidades, efeitos e custo do cuidado prestado); \\
Legitimidade & Conformidade com preferências sociais em relação a tudo mencionado acima; \\
\hline Equidade & Igualdade na distribuição do cuidado e de seus efeitos sobre a saúde. \\
\hline
\end{tabular}

Fonte: Donabedian (1990) ${ }^{18}$ 
proposto por Starfield (2001), que apresenta parâmetros universais de avaliação. Focado na comunidade, nos pacientes e nos profissionais de saúde, é um instrumento que avalia as características nucleares da APS: acessibilidade, integralidade, longitudinalidade e coordenação, além de oferecer informaçóes acerca dos aspectos derivativos da Atenção Primária: centralidade na família, orientação comunitária e competência cultural ${ }^{4}$.

O PCA-Tool tem a vantagem de ter componentes específicos para ciclos de vida e de permitir a comparaçáo das equipes de Atenção Primária brasileiras com equipes de todo o mundo ${ }^{23,24,25}$.

Por fim, com a mudança de comando no Ministério da Saúde, uma série de portarias define novas políticas, incluindo uma nova Política Nacional da Atenção Básica, e novos formatos de incentivos, dentre eles um novo mecanismo de avaliação da qualidade. O PMAQ-AB utiliza 47 indicadores de produção ambulatorial e cadastramento, relacionados a sete áreas estratégicas: Saúde da Mulher, Saúde da Criança, Controle de Diabetes Mellitus e Hipertensão Arterial Sistêmica, Saúde Bucal, Produçáo Geral, Tuberculose e Hanseníase, e Saúde Mental. Suas inovaçóes em relação a modelos anteriores são:

- o uso de indicadores de desempenho para classificar equipes, e indicadores de monitoramento sobre a oferta de serviços e resultados alcançados por equipe;

- repasse imediato e automático após contratualização, com recebimento de recursos durante até seis meses independente da qualidade antes da avaliação externa;

- se destinar a qualquer equipe de saúde da atenção básica, independente do modelo adotado; e

- agrupar municípios sob avaliação, para fins de comparação, em seis estratos de acordo com indicadores socioeconômicos como Produto Interno Bruto per capita, proporção populacional de acesso a planos de saúde, bolsa família e extrema pobreza, e densidade demográfica.

Cabe ressaltar que não há dentre os 47 indicadores do PMAQ-AB algum voltado especificamente para avaliar a qualidade da gestáo. Esta será realizada por avaliadores externos, com parâmetros de avaliação da gestão municipal e da coordenação da atenção básica, não especificados nesta portaria, e com critérios locais voltados para infraestrutura e insumos básicos das Unidades de Saúde, sem previsão de avaliação do gestor destas Unidades ${ }^{6}$.

\section{Conclusão}

Toda mudança de processo de gestáo interfere na cultura de uma organização, especialmente quando direcionada para o tema da qualidade. E a mudança da cultura organizacional envolverá todos os processos, meios internos, e a forma como o usuário (consumidor) a vê e se relaciona com ela. Ela não pode ser artificialmente produzida por portarias, e suas mudanças náo ocorrem sem quebra de paradigmas.

Uma mudança organizacional na saúde envolve a modificação do foco na cura e reabilitação baseadas em tecnologias medicocentradas, para um foco na promoçáo da saúde e prevenção baseada em evidências, apoiadas no resgate da relação profissional-paciente e em tecnologias leves e multiprofissionais. Este desafio reflete a mudança na forma de remuneração de médicos e equipes, do pagamento por procedimentos ou por salários fixos para o pagamento por performance ou desempenho.

Especialmente no que se refere à saúde pública, envolve também uma mudança na qualidade dos gestores do SUS, na relação do gestor com os profissionais de saúde e usuários, e na participação ativa dos profissionais da saúde - também usuários, clientes e público-alvo dos serviços do SUS - na pactuação destas metas e parâmetros de qualidade. A qualificação da gestão em todos os seus níveis, em especial no local, é um passo a ser dado e deve ser igualmente avaliada em termos de práticas e posturas.

Não se melhora a qualidade pela produtividade. É necessário estruturar um sistema gerencial profissional que coordene os instrumentos disponíveis e garanta o planejamento, controle e melhorias de cada um dos processos, e que aplique o conceito de cadeia de valor: uma sequência de processos de produçáo relacionados entre si, dos insumos (recursos humanos, materiais e financeiros) à produção de serviços até a distribuição dos serviços ao usuário. Em outras palavras: a rede de saúde deve funcionar como equipe.

Será importante confirmar a satisfação do usuário com a qualidade da Estratégia Saúde da Família como um dos componentes da PMAQ-AB. Mas náo basta estabelecer um sistema de avaliação da qualidade de serviços. Há que se investir fortemente na qualificação dos profissionais que trabalham nestas instituiçóes, na recompensa para aqueles profissionais que realmente consigam imprimir qualidade no serviço, e na ambientação destes serviços.

\section{Referências}

1. Stachelski L. O impacto da implantação da estratégia da Gestão da Qualidade Total na Cultura Organizacional: um estudo de caso. [Dissertação] Florianópolis: Universidade Federal de Santa Catarina; 2001. 137 p.

2. Teixeira MHWR. Gestão da qualidade em serviços de saúde: a utilização do método gerencial TQC. [Monografia]. Rio de Janeiro: Universidade Gama Filho, Universidade Unimed; 2003. 132 p. 
3. Sampaio SL. A Cultura Organizacional na Gestão da Qualidade. [Monografia]. Belo Horizonte: Universidade Unimed; 2011. 26 p.

4. Starfield B. Atenção Primária: equilíbrio entre necessidades de saúde, serviços e tecnologia. Brasília: UNESCO, Ministério da Saúde; 2002. 726 p.

5. Minas Gerais. Secretaria de Estado de Saúde. Pacto de gestão: da municipalização autárquica à regionalização cooperativa. Belo Horizonte: SESMG; 2004. 80 p.

6. Brasil. Ministério da Saúde. Portaria n 1.654, de 19 de julho de 2011. Institui, no âmbito do Sistema Único de Saúde, o Programa Nacional de Melhoria do Acesso e da Qualidade da Atenção Básica (PMAQ-AB) e o Incentivo Financeiro do PMAQ-AB, denominado Componente de Qualidade do Piso de Atenção Básica Variável - PAB Variável. Diário Oficial da República Federativa do Brasil, Brasília, DF, 20 jul. 2011. Sessão 1, n. 138, p. 79

7. Minas Gerais. Escola de Saúde Pública do Estado de Minas Gerais - ESPMG. Implantação do Plano Diretor da Atenção Primária à Saúde: Redes de Atenção à Saúde. Belo Horizonte: ESPMG; 2008.

8. Savassi LCM. Iniciação a prática de Saúde da Família. Belo Horizonte: Faculdade Senac (Especialização em Saúde da Família); 2011. 80 p. Apostila.

9. Mendes EV. As Redes de Atenção à Saúde. RMMG. 2008; 18(4-S4).

10. Fundação Oswaldo Cruz - Fiocruz. Escola Nacional de Saúde Pública.. Rev Radis. 2011; (109):12-18.

11. Zucchi P, Del Nero C, Malik AM. Gastos em saúde: os fatores que agem na demanda e na oferta dos serviços de saúde. Rev Saude Soc. 2000; 9(1-2):127-150. http://dx.doi.org/10.1590/S010412902000000100010

12. Tavares-Júnior FA. Os instrumentos de contratualização e a pactuação por resultados. In: II Congresso Consad de Gestão Pública - Painel 9: Inovações no sistema de gestão do Sistema Único de Saúde (SUS); 2009, Brasília. [online]. CONSAD; 2009. [acesso em 01/04/2011]. Disponível em: http://www.seplag.rs.gov.br/uploads/ Painel_09_Francisco_Tavares_formatado.pdf

13. Mendes EV. As redes de atenção à saúde. Ciênc Saúde Coletiva. 2010; 15(5):2297-2305. http://dx.doi.org/10.1590/S141381232010000500005

14. Savassi LCM. A satisfação do usuário e a autopercepção da saúde em atenção primária. Rev Bras Med Fam Comunidade. 2010; 5(17):3-5.
15. Instituto de Pesquisa Econômica Aplicada - IPEA. Sistema de Indicadores de Percepção Social Saúde. Brasília: IPEA; 2011. 20 p. Disponível em: http://www.ipea.gov.br/portal/images/stories/PDFs/ SIPS/110207_sipssaude.pdf

16. Brasil. Ministério da Educação. Ministério da Saúde. Portaria interministerial $n^{\circ} 2.087$, de 01 de setembro de 2011. Institui o Programa de Valorização do Profissional da Atenção Básica. Diário Oficial da República Federativa do Brasil, Brasília, DF, 02 set. 2011.

17. Brasil. Ministério da Saúde. Portaria $n^{\circ} 2.488$, de 21 de outubro de 2011. Aprova a Política Nacional de Atenção Básica, estabelecendo a revisão de diretrizes e normas para a organização da Atenção Básica, para a Estratégia Saúde da Família (ESF) e o Programa de Agentes Comunitários de Saúde (PACS). Diário Oficial da República Federativa do Brasil, Brasília, DF, 24 out. 2011.

18. Donabedian A. The seven pillars of quality. Arch Pathol Lab Med.1990; 114:1115-1118.

19. Bueno H. A Utilização da Sala de Situação de Saúde no Distrito Federal. Brasília: Universidade de Brasília; 2003. 109 p.

20. Brasil. Ministério da Saúde. Rede Nacional de Informações em Saúde. Sala de Situação. [website] [online] [acesso em 26/12/2011]. Disponível em: http://www.datasus.gov.br/rnis/apresentacao.htm]

21. Brasil. Ministério da Saúde, Secretaria de Atenção a Saúde. Departamento de Atenção Básica. Avaliação para a melhoria da qualidade da estratégia saúde da família. Brasília: Ministério da Saúde; 2005. 6 v.

22. Brasil. Ministério da Saúde, Secretaria de Atenção a Saúde. Departamento de Atenção Básica. Prograb: Programação de gestão por resultados. Brasília: Ministério da Saúde; 2006. 160 p.

23. Starfield B, Xu J, Shi L. Validating the Adult Primary Care Assessment Tool. J Fam Pract. 2001; 50(2):161-175.

24. Harzheim E, Starfield B, Rajmil L, Álvarez-Dardet C, Stein AT. Consistência interna e confiabilidade da versão em português do Instrumento de Avaliação da Atenção Primária (PCATool-Brasil) para serviços de saúde infantil. Cad Saúde Pública. 2006; 22(8):1649-1659. http://dx.doi.org/10.1590/S0102-311X2006000800013

25. Brasil. Ministério da Saúde. Secretaria de Atenção em Saúde. Departamento de Atenção Básica. Manual do instrumento de avaliação da atenção primária à saúde: primary care assessment tool PCATool. Brasilia: Ministério da Saúde; 2010. 80 p. 